\title{
Pasta CTZ para abordaje endodóncico de dientes primarios: Una revisión narrativa de la literatura
}

\author{
Ana Paula Portes Zeno, ${ }^{1}$ (D) Guido A Marañon-Vásquez, ${ }^{2}$ Laura Guimarães Primo, ${ }^{3}$ \\ Andréa Vaz Braga Pintor, ${ }^{4}$ Marcelo de Castro Costa. ${ }^{5}$
}

\begin{abstract}
Resumen: Introducción: La afectación pulpar irreversible de los dientes primarios representa un desafío para la endodoncia en odontopediatría. Sobre la base del enfoque de esterilización de lesiones y reparación de tejidos (LSTRLesion Sterelization and Tissue Repair), se ha propuesto una técnica endodóncica no instrumental que utiliza pastas antibióticas. La pasta antibiótica CTZ, que contiene una mezcla de cloranfenicol, tetraciclina, óxido de zinc y eugenol, tiene potencial para uso en la técnica endodóncica no instrumental en pacientes pediátricos. Objetivo: El objetivo de este trabajo es presentar una revisión narrativa de la literatura sobre la pasta antibiótica CTZ, en lo relacionado a capacidad antimicrobiana, biocompatibilidad y aspectos clínicos. Materiales y métodos: Se realizaron búsquedas en las bases de datos PUBMED, BVS, WEB OF SCIENCE, COCHRANE y SCOPUS utilizando los términos "ctz paste", "ctz", "tooth, deciduous" y "primary molars" sin restricción de idioma, con fecha limitada de 2015 hasta enero de 2021. Resultados: Después de eliminar los duplicados, se obtuvieron 13 artículos. La pasta CTZ mostró una actividad antimicrobiana satisfactoria en Enterecoccus faecalis, Staphylococcus aureus, Klebisiella pneumonia, Escherichia coli y Candida albicans. Los estudios in vitro e in vivo han demostrado que la pasta tiene biocompatibilidad. La frecuencia de éxito clínico varió del $37 \%$ al $100 \%$ y el éxito radiográfico del $29,7 \%$ al 97,4\%. Conclusiones: Aunque se necesitan más estudios que contribuyan a un mejor nivel de la evidencia, puede afirmase que la pasta CTZ una es alternativa para los casos de afectación pulpar en dientes primarios, inclusive en el ámbito de la salud pública.
\end{abstract}

Palabras clave: diente primario, endodoncia, antibacterianos, ensayo de materiales, ensayo clínico.

\section{Pasta CTZ na abordagem endodôntica de dentes decíduos: uma revisão de literatura}

Resumo: Introdução: O envolvimento pulpar irreversível dos dentes decíduos, representa um desafio à endodontia em odontopediatria. Com base na abordagem de esterilização da lesão e reparo tecidual (LSTR- Lesion Sterelization and Tissue Repair), a técnica endodôntica não instrumental com utilização de pastas antibióticas tem sido proposta. A pasta antibiótica CTZ, que contém uma mistura de cloranfenicol, tetraciclina, óxido de zinco e eugenol, apresenta potencial para uso na técnica endodôntica não instrumental em pacientes pediátricos. Objetivo: O objetivo deste trabalho é apresentar uma revisão narrativa da literatura sobre a pasta antibiótica CTZ, abrangendo capacidade antimicrobiana, biocompatibilidade e aspectos clínicos. Materiais e métodos: Foi realizada busca nas bases de dados PUBMED, BVS, WEB OF SCIENCE, COCHRANE e SCOPUS usando os termos "ctz paste", "ctz", "tooth, deciduous" e "primary molars" com data limitada de 2015 a janeiro de 2021. Resultados: Após a remoção de duplicatas 13 artigos foram obtidos. A pasta CTZ mostrou atividade antimicrobiana satisfatória sobre Enterecoccus faecalis, Staphylococcus aureus, Klebisiella pneumonia, Escherichia coli y Candida albicans. Estudos in vitro e in vivo demonstraram que a pasta apresenta boa biocompatibilidade. A frequência de sucesso clínico variou de $37 \%$ a $100 \%$ e o sucesso radiográfico de $29,7 \%$ a $97,4 \%$. Conclusão: Embora mais estudos que contribuam para um melhor nível de evidência sejam necessários, a pasta antibiótica CTZ apresenta atividade antimicrobiana, biocompatibilidade e resultados clínicos satisfatórios, podendo ser uma alternativa interessante para os casos de comprometimento pulpar de dentes decíduos, inclusive em saúde pública.

Palabra-chave: dente decíduo, endodontia, antibacterianos, teste de materiais, ensaio clínico.

\footnotetext{
1 Alumna de la Maestría Profesional en Clínica Odontológica, Facultad de Odontología, Universidad Federal de Río de Janeiro.

2 Alumno de el Doctorado, Departamento de Odontopediatría y Ortodoncia, Facultad de Odontología, Universidad Federal de Río de Janeiro.

$3 \mathrm{PhD}$. Profesora investigadora y Docente del programa de Maestría Profesional en Clínica Odontológica y del área de Odontopediatria, Departamento de Odontopediatria y Ortodoncia, Facultad de Odontología, Universidad Federal de Rio de Janeiro.

4 PhD. Profesora investigadora y Docente del área de Odontopediatría, Departamento de Odontopediatría y Ortodoncia, Facultad de Odontología, Universidad Federal de Río de Janeiro.

PhD. Profesor investigador y Docente del área de Odontopediatría, Departamento de Odontopediatría y Ortodoncia, Facultad de Odontología, Universidad Federal de Río de Janeiro.
} 


\section{CTZ paste for endodontic approach to deciduous teeth: A literature review}

Abstract: Introduction: The irreversible pulp changes in primary teeth represent a challenge to endodontics in pediatric dentistry. Based on the Lesion Sterilization and Tissue Repair approach (LSTR), a non-instrumental endodontic treatment (NIET) of primary teeth root canals using an antibiotic paste has been proposed. The CTZ antibiotic paste, which contains a mixture of chloramphenicol, tetracycline, zinc oxide and eugenol, has potential for use in pediatric patients. Objective: The objective of this work is to present a literature review on the CTZ antibiotic paste, covering antimicrobial capacity, biocompatibility, and clinical aspects. Methods: An electronic search on PUBMED, BVS, WEB OF SCIENCE, COCHRANE y SCOPUS databases using the terms "ctz paste", "ctz", "deciduous teeth" and "primary molars" without language restrictions and publication date between 2015 and January 2021. Results: After removing duplicates, a total of 13 articles were included. The CTZ paste showed satisfactory antimicrobial activity on Enterecoccus faecalis, Staphylococcus aureus, Klebisiella pneumonia, Escherichia coli and Candida albicans. In vivo and in vitro studies have demonstrated that the paste presents good biocompatibility. The frequency of clinical success varied from $37 \%$ to $100 \%$ and radiographic success from $29,7 \%$ to $97,4 \%$. Conclusion: Although more studies are needed to contribute to the body of evidence, the CTZ antibiotic paste demonstrated antimicrobial activity, biocompatibility and satisfactory clinical results and may be an alternative for cases of pulp involved primary teeth, including in public health.

Key words: tooth, deciduous, endodontics, anti-bacterial agents, materials testing, clinical trial.

\section{Introducción}

La afectación pulpar de los dientes primarios ya sea por caries o traumatismo dental, representa un reto para el tratamiento endodóncico en odontopediatría, debido a la compleja anatomía de estos dientes, escasa cooperación de los pacientes infantiles, factores que dificultan el intento por neutralizar o reducir los microorganismos en el sistema de conductos radiculares. ${ }^{1}$

El enfoque de esterilización de lesiones y reparación de tejidos (LSTR - Lesion Sterilization and Tissue Repair) propuesto en la Universidad de Niigata, ${ }^{2}$ se basa en el concepto de que existe la posibilidad de reparación de los tejidos dañados una vez que estos son desinfectados. Siguiendo este abordaje, fue propuesta una técnica sencilla, sin instrumentación convencional de los conductos radiculares, con el uso de una pasta antibiótica en la entrada de los conductos radiculares para el tratamiento de dientes primarios con daño pulpar irreversible. La pasta antibiótica CTZ, que contiene una mezcla de cloranfenicol, tetraciclina, óxido de zinc y eugenol, fue propuesta en 1965 y 1967 por Cappiello ${ }^{3,4}$ para este tratamiento, ha sido utilizada en América Latina a pesar de la limitada cantidad de estudios clínicos. ${ }^{5-10}$ Esta pasta tiene la ventaja de ser de fácil y rápido uso, en una sola sesión clínica. Sin embargo, los resultados reportados en la literatura sobre el éxito clínico de esta intervención no se han mostrado tan favorables durante la monitorización radiográfica de las lesiones. ${ }^{5}$ Aunque en una revisión sistemática al comparar estudios realizados con diferentes versiones de la pasta $3 \mathrm{Mix}$, con y sin tetraciclinas, se observó una menor tasa de éxito para las formulaciones sin tetraciclina, la certeza de la evidencia fue categorizada como muy baja, debido a problemas muy graves de heterogeneidad y comparación indirecta. ${ }^{11}$ Entonces, si bien no hay evidencia directa con respecto a la pasta 
CTZ, el objetivo de esta revisión narrativa fue presentar la literatura reciente sobre la pasta antibiótica CTZ en relación a su actividad antimicrobiana, biocompatibilidad y tasa de éxito clínico y radiográfico, con el fin de contribuir al conocimiento sobre el potencial uso de este material para el tratamiento endodóncico de dientes primarios con daño pulpar irreversible.

\section{Materiales y métodos}

Para elaboración de esta revisión narrativa, se realizaron búsquedas electrónicas en las bases de datos PUBMED, BVS, WEB OF SCIENCE, COCHRANE y SCOPUS utilizando los términos "ctz paste", "ctz", "tooth deciduous" y "primary molars". Los criterios de inclusión para la selección de los estudios fueron: estudios clínicos realizados en molares primarios con diagnóstico de necrosis pulpar o pulpitis irreversible, tratados con técnica LSTR y pasta CTZ, con un seguimiento clínico y radiográfico mínimo de 6 meses y estudios de laboratorio que evaluaran la biocompatibilidad y actividad antimicrobiana de la pasta CTZ, publicados entre 2015 y Enero de 2021, en inglés, español o portugués. Fueron excluidos del estudio: reportes de caso, disertaciones, tesis y estudios que no contemplaron los aspectos propuestos. Dos evaluadores (APPMZ y AVBP) realizaron la selección de los estudios.

\section{Resultados}

Tras eliminar los duplicados y las tesis, y leer los resúmenes, se recuperaron trece artículos que cubrían los temas de actividad antimicrobiana, ${ }^{12-15}$ biocompatibilidad ${ }^{16-18}$ y aspectos clínicos ${ }^{5-10}$ del uso de la pasta CTZ en el tratamiento endodóncico de los dientes primarios (Figura 1).

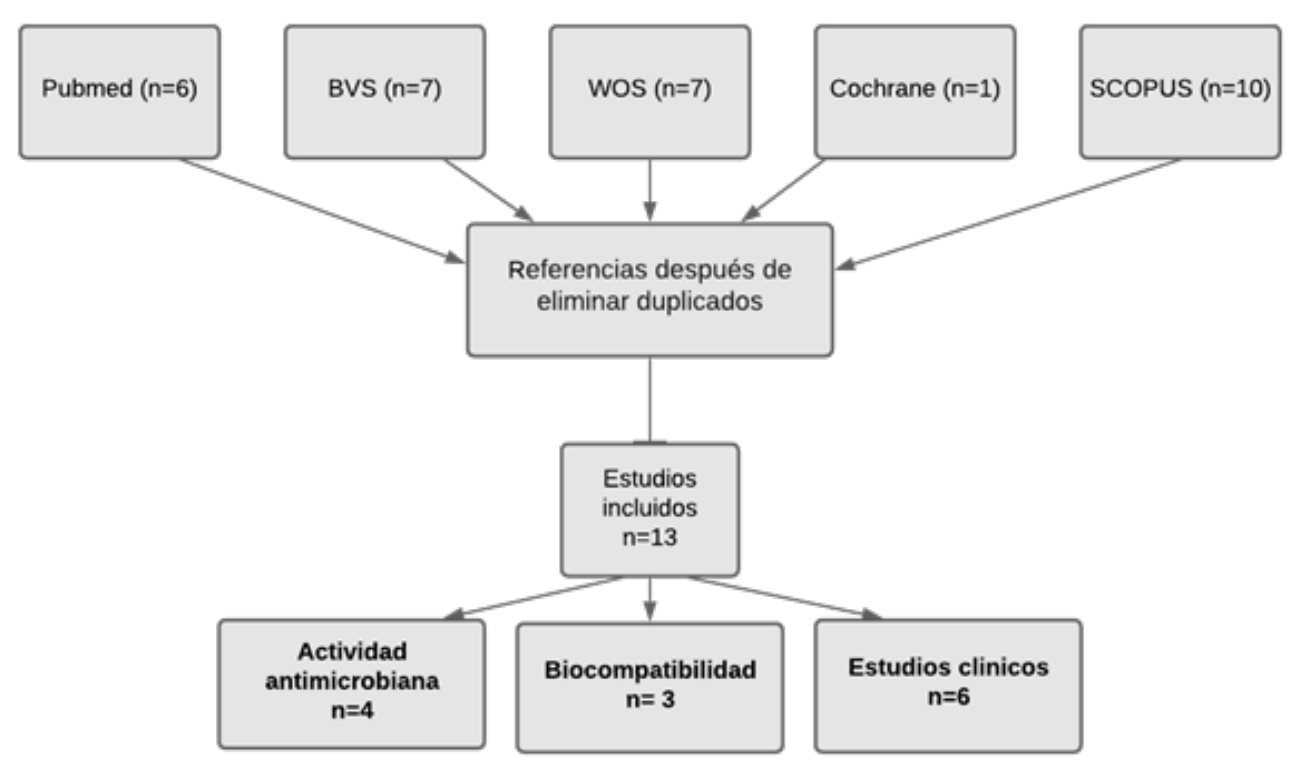

Figura 1. Diagrama de flujo. 


\section{Actividad antimicrobiana}

Las características de los estudios sobre la actividad antimicrobiana de la pasta CTZ se resumen en la tabla 1 . Se obtuvieron cuatro estudios de laboratorio utilizando diferentes metodologías: difusión en agar ${ }^{12,13}$ y contacto directo. ${ }^{14,15}$ La pasta CTZ mostró una actividad antimicrobiana satisfactoria en Enterecoccus faecalis, Staphylococcus aureus, Klebisiella pneumonia, Escherichia coli y Candida albicans.

\section{Biocompatibilidad}

En los estudios incluidos, para evaluar la biocompatibilidad de la pasta CTZ en comparación con materiales para uso endodóncico, se utilizaron diferentes diseños de estudio: estudio en animales con implantes subcutáneos y observación de la reacción tisular local, ${ }^{16}$ estudio clínico en humanos con análisis histológico de tejidos ${ }^{17}$ y ensayo citotóxico in vitro utilizando cultura celular. $^{18}$

La tabla 2 resume las características de los tres estudios incluidos con relación al diseño del estudio, objetivo, materiales probados, sistema de prueba, principales resultados y conclusiones.

\section{Estudios clínicos}

Las principales características de los estudios clínicos incluidos se describen en la tabla 3.

Se obtuvieron seis estudios: cuatro ensayos clínicos aleatorizados, ${ }^{5,6,8,9}$ una serie de $\operatorname{casos}^{7}$ y un estudio observacional transversal. ${ }^{10}$ Los estudios fueron conducidos en países de América Latina con la excepción de uno, originario de la India. ${ }^{9}$
El tiempo mínimo de seguimiento fue de seis meses y el máximo de treinta y seis meses.

En la mayoría de los estudios experimentales, el aislamiento absoluto se realizó durante el tratamiento y en sólo un estudio, se utilizó el aislamiento relativo. ${ }^{6}$

Respecto al diagnóstico pulpar inicial, dos estudios eligieron dientes con indicación de pulpectomía por signos y síntomas de pulpitis irreversible o necrosis pulpar, 5,9 un estudio incluyó dientes con pulpas vitales sin signos de degeneración, sin sintomatología dolorosa y que sufrieron exposición por caries ${ }^{8}$ y tres estudios eligieron solamente casos de necrosis pulpar. $6,7,10$

Los materiales de elección para la restauración dental final fueron coronas de acero, ${ }^{8,9}$ cemento de ionómero de vidrio ${ }^{6}$ o resina compuesta fotopolimerizable. ${ }^{5}$

En todos los estudios se realizó la técnica no instrumental propuesta en la descripción del concepto LSTR, donde se debe realizar una intervención mínima. ${ }^{2}$

Tres ensayos clínicos aleatorizados, ${ }^{6,8,9}$ obtuvieron tasas de éxito clínico satisfactorias para la pasta CTZ, con mejores resultados clínicos que radiográficos. Solo un estudio calificó la técnica de uso de la pasta CTZ como insatisfactoria. ${ }^{5}$ Tasa de éxito clínico y radiográfico satisfactorias fue reportado en el estudio serie de $\operatorname{casos}^{7}$ (Tabla 3). 


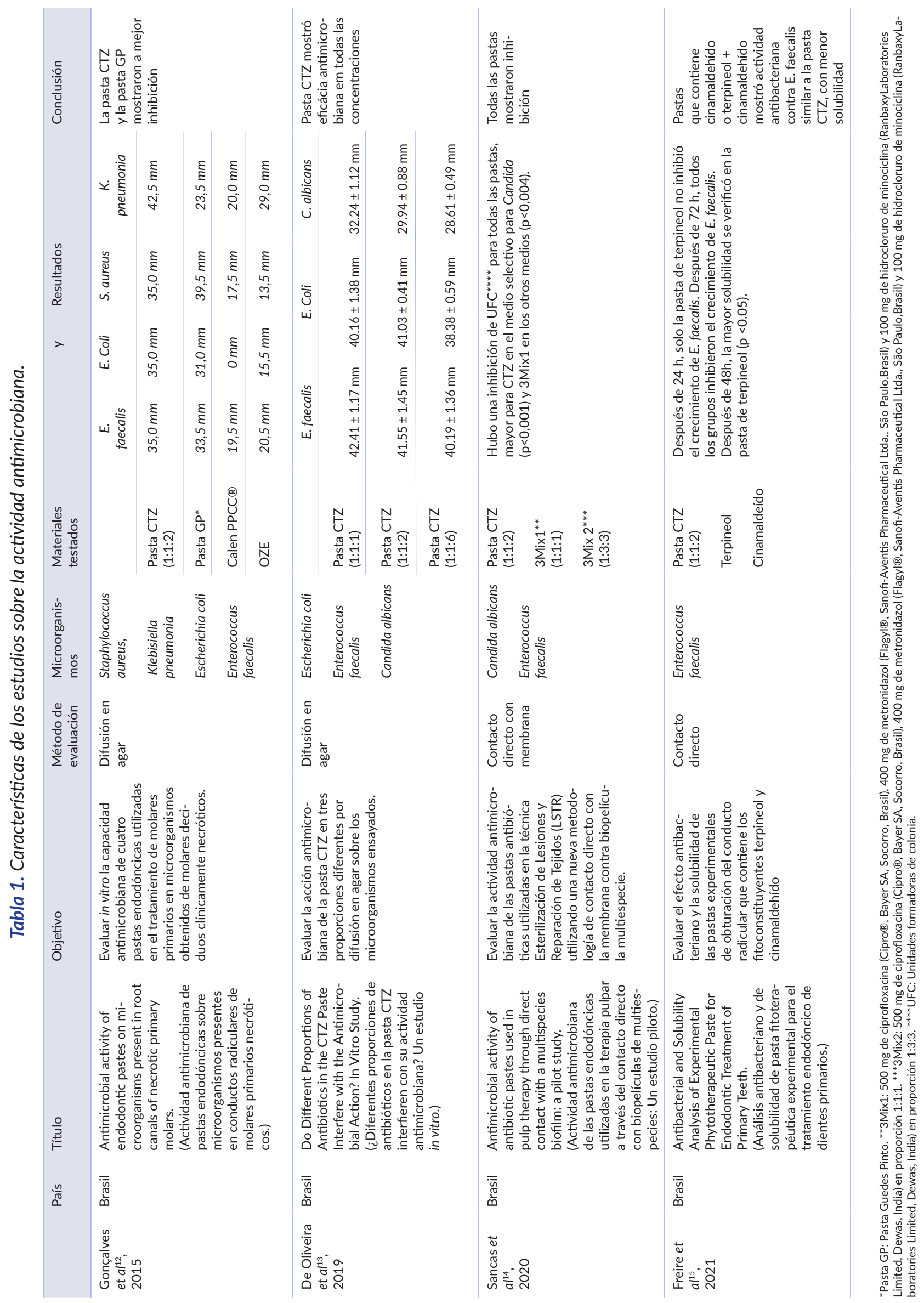




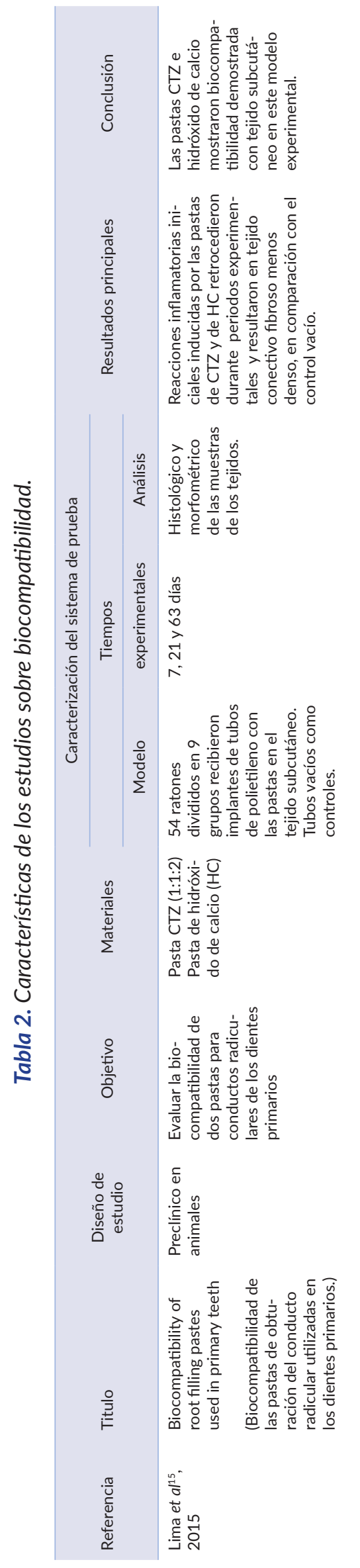

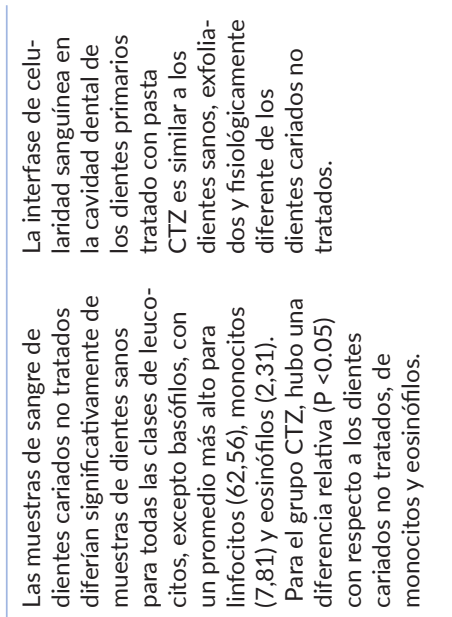

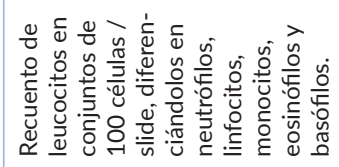

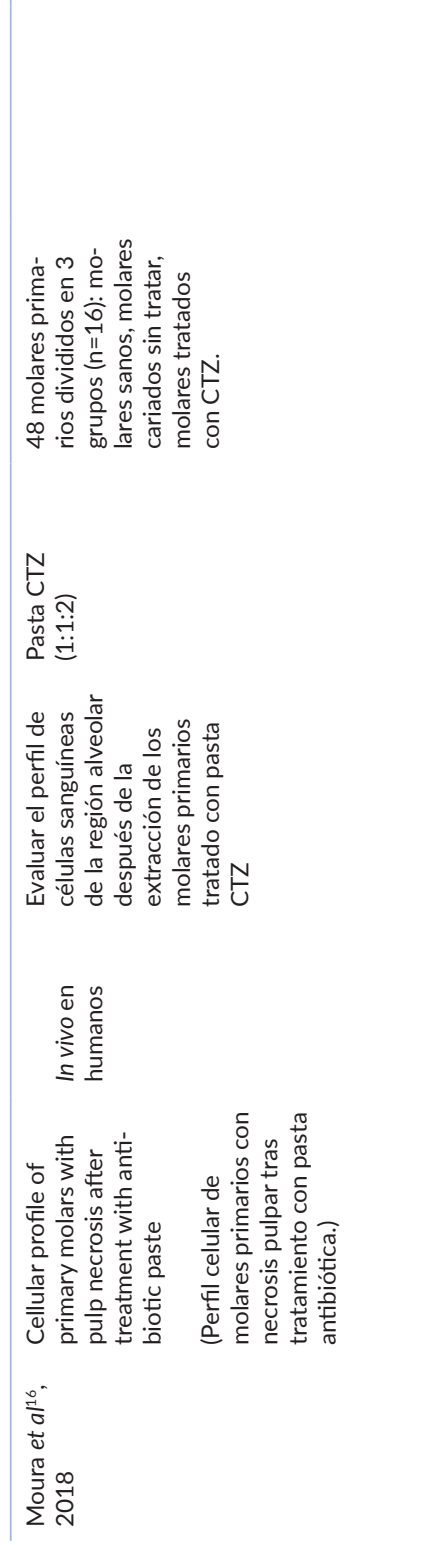

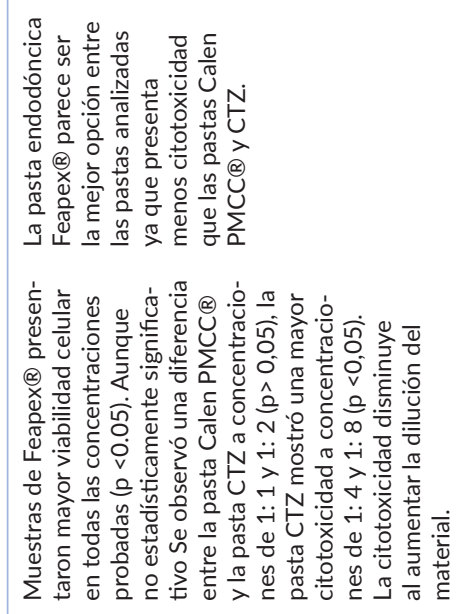

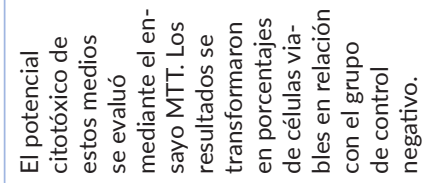

$\frac{n}{\sqrt[n]{0}}$

热

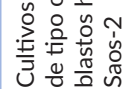

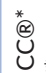

$\sum_{0} \stackrel{*}{*} \times$

Uั

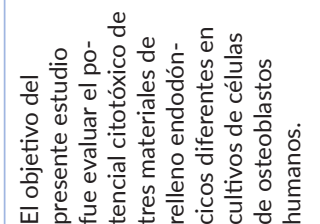

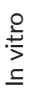
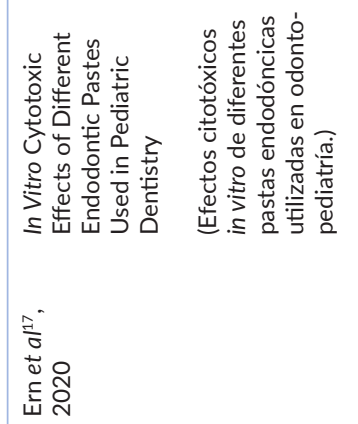

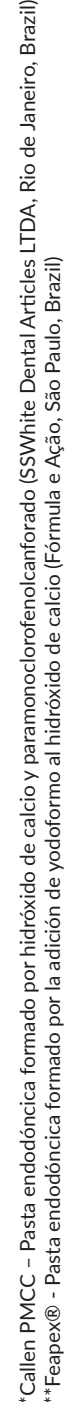

Vol $12 \mathrm{~N}^{\circ} 1$ Enero - Junio 2022 
Tabla 3. Principales características de los estudios clínicos incluidos

\begin{tabular}{|c|c|c|c|c|c|c|}
\hline Referencia & Pais & Título & $\begin{array}{l}\text { Diseño de } \\
\text { Estudio }\end{array}$ & Objetivo & Principales Resultados & Conclusiones \\
\hline $\begin{array}{l}\text { Daher et } a l^{5} \text {, } \\
2015\end{array}$ & Brasil & $\begin{array}{l}\text { Ineffectiveness of Anti- } \\
\text { biotic-Based Pulpotomy } \\
\text { for Primary Molars: A } \\
\text { Survival Analysis. } \\
\text { (Ineficacia de la pulpo- } \\
\text { tomía con antibióticos } \\
\text { para los molares } \\
\text { primarios: a } \\
\text { Análisis de supervi- } \\
\text { vencia). }\end{array}$ & $\begin{array}{l}\text { Ensayo clínico } \\
\text { aleatorizado }\end{array}$ & $\begin{array}{l}\text { Investigar la tasa de super- } \\
\text { vivencia a dos años de los } \\
\text { molares primarios tratados } \\
\text { con tratamiento endodónci- } \\
\text { co sin instrumentación con } \\
\text { pasta antibiótica mixta que } \\
\text { contiene } \\
\text { cloranfenicol, tetraciclina, } \\
\text { óxido de zinc y eugenol (CT- } \\
\text { Z)-Grupo I y compárelo } \\
\text { al tratamiento de conducto } \\
\text { convencional con pasta de } \\
\text { hidróxido de calcio-Grupo II. }\end{array}$ & $\begin{array}{l}\text { Los niños fueron seguidos } \\
\text { durante } 1 \text { a } 26 \text { meses. } \\
\text { Las tasas de fracaso del trata- } \\
\text { miento fueron: } \\
73,0 \% \text { en el Grupo I(CTZ) y } \\
31,3 \% \text { en el Grupo II }\left(\mathrm{HC}^{*}\right) \\
\text { El grupo I (media } 13,2 \text {; IC del } \\
95 \% \text { : } 10,2-16,3) \text { tuvo un me- } \\
\text { nor tasa de supervivencia que } \\
\text { el Grupo II (media } 18,9 ; \text { IC del } \\
95 \%: 14,5-23,2)(p=0,02)\end{array}$ & $\begin{array}{l}\text { Tratamiento endodóncico sin } \\
\text { instrumentación de molares } \\
\text { primarios con la pasta CTZ } \\
\text { resultó en una baja tasa de } \\
\text { supervivencia en un segui- } \\
\text { miento de dos años; el fracaso } \\
\text { radiográfico desalienta su uso } \\
\text { en lugar de endodoncia con- } \\
\text { vencional de canal radicular. }\end{array}$ \\
\hline $\begin{array}{l}\text { Siegl et al } \\
2015\end{array}$ & Brasil & $\begin{array}{l}\text { Two endodontics } \\
\text { techniques analysis in } \\
\text { primary molars with } \\
\text { fistula. } \\
\text { (Análisis de dos } \\
\text { técnicas endodóncicas } \\
\text { en molares primarios } \\
\text { fistulados.) }\end{array}$ & $\begin{array}{l}\text { Ensayo clínico } \\
\text { aleatorizado }\end{array}$ & $\begin{array}{l}\text { La propuesta de investi- } \\
\text { gación fue monitorear el } \\
\text { área radiolúcida (píxeles) } \\
\text { en la región de furca y la } \\
\text { respuesta clínica de los } \\
\text { molares primarios tratados } \\
\text { endodoncicamente con dife- } \\
\text { rentes técnicas simplificadas } \\
\text { (tratamiento endodóncico sin } \\
\text { instrumentación): Pasta CTZ } \\
\text { y pasta Guedes Pinto. }\end{array}$ & $\begin{array}{l}\text { La reducción en el área radio- } \\
\text { lúcida inicial después de } 6 \text { ( } p \\
<0.01) \text { y } 12 \text { meses }(p<0.01) \text { se } \\
\text { observó solo con el uso de la } \\
\text { pasta CTZ. El uso de pasta } \\
\text { Guedes no resultó en una } \\
\text { disminución en el área radiolú- } \\
\text { cida después de } 12 \text { meses de } \\
\text { seguimiento }(p=0,09) \text {. }\end{array}$ & $\begin{array}{l}\text { La respuesta clínica fue similar } \\
\text { en ambos grupos, pero solo los } \\
\text { dientes tratados con la pasta } \\
\text { CTZ mostraron una reducción } \\
\text { en el área radiotransparente en } \\
\text { la región de furca a los } 6 \text { y } 12 \\
\text { meses de seguimiento }\end{array}$ \\
\hline $\begin{array}{l}\text { De Deus } \\
\text { Moura et } a l^{7} \\
2016\end{array}$ & Brasil & $\begin{array}{l}\text { Endodontic Treatment } \\
\text { of Primary Molars with } \\
\text { Antibiotic Paste: A } \\
\text { Report of } 38 \text { Cases. } \\
\text { (Tratamiento endo- } \\
\text { dóncico de molares } \\
\text { primarios con pasta } \\
\text { antibiótica: A } \\
\text { Informe de } 38 \text { casos.) }\end{array}$ & Serie de casos & $\begin{array}{l}\text { Presentar } 38 \text { casos de } \\
\text { molares primarios con pulpas } \\
\text { necróticas tratados con pasta } \\
\text { antibiótica CTZ. }\end{array}$ & $\begin{array}{l}\text { Para los } 38 \text { dientes que se } \\
\text { habían presentado con un abs- } \\
\text { ceso, dolor o movilidad patoló- } \\
\text { gica, los datos indicaron que la } \\
\text { endodoncia adoptada los tra- } \\
\text { tamientos mostraron un éxito } \\
\text { clínico del } 100 \% \text {. Dentro de } \\
\text { parámetros de éxito, } 95,8 \% \text {, } \\
100 \%, 93,5 \% \text { y } 93,5 \% \text { de los } \\
\text { molares primarios mandibu- } \\
\text { lares tratados con pasta CTZ } \\
\text { mostraron éxito radiográfico } \\
\text { en los tiempos de evaluación } \\
\text { de } 3-6,7-12,13-24 \text { y } 25-36 \\
\text { meses, respectivamente }\end{array}$ & $\begin{array}{l}\text { El tratamiento endodóncico de } \\
\text { molares primarios mandibu- } \\
\text { lares con pulpas necróticas } \\
\text { utilizando la pasta CTZ, mostró } \\
\text { resultados clínicos excelentes } \\
\text { y buenos resultados radiográ- } \\
\text { ficos. }\end{array}$ \\
\hline $\begin{array}{l}\text { Luengo et } a l^{8} \text {, } \\
2019\end{array}$ & México & $\begin{array}{l}\text { Clinical and Radio- } \\
\text { graphic Evaluation } \\
\text { of Formocresol and } \\
\text { Chloramphenicol, } \\
\text { Tetracycline and Zinc } \\
\text { Oxide-Eugenol Anti- } \\
\text { biotic Paste in Primary } \\
\text { Teeth Pulpotomies: } 24 \\
\text { month follow up. } \\
\text { (Evaluación clínica } \\
\text { y radiográfica de } \\
\text { formocresol y pasta an- } \\
\text { tibiótica de cloranfeni- } \\
\text { col, tetraciclina y óxido } \\
\text { de zinc-eugenol en } \\
\text { pulpotomías de dientes } \\
\text { primarios: seguimiento } \\
\text { de } 24 \text { meses.) }\end{array}$ & $\begin{array}{l}\text { Ensayo clínico } \\
\text { aleatorizado }\end{array}$ & $\begin{array}{l}\text { Evaluar clínica y radiográ- } \\
\text { ficamente la efectividad } \\
\text { de formocresol y la pasta } \\
\text { antibiótica CTZ (cloranfeni- } \\
\text { col, tetraciclina y óxido de } \\
\text { zinc-eugenol) en pulpotomías } \\
\text { de dientes primarios, durante } \\
\text { un período de } 6,12 \text { y } 24 \\
\text { meses. }\end{array}$ & $\begin{array}{l}\text { Después de } 24 \text { meses de se- } \\
\text { guimiento se obtuvo un éxito } \\
\text { clínico del } 100 \% \text { y del } 94,3 \% \text {, } \\
\text { en los grupos CTZ y formocre- } \\
\text { sol respectivamente } \\
\left(x^{2}=0,450, p>0,05\right) \text {. } \\
\text { El éxito radiográfico fue del } \\
97,4 \% \text { y } 94,3 \% \text { respectiva- } \\
\left.\text { mente ( } x^{2}=0,920, p>0,05\right) \text {. }\end{array}$ & $\begin{array}{l}\text { El rendimiento de la pasta } \\
\text { antibiótica CTZ fue superior al } \\
\text { del formocresol. No } \\
\text { se observaron diferencias } \\
\text { estadísticamente significativas } \\
\text { entre los grupos de tratamien- } \\
\text { to, ya sea clínica o radiográfi- } \\
\text { camente. } \\
\text { Se deben realizar más ensayos } \\
\text { clínicos aleatorios antes de } \\
\text { que pueda indicarse de forma } \\
\text { segura. }\end{array}$ \\
\hline
\end{tabular}


Tabla 3. Principales características de los estudios clínicos incluidos. (cont.)

\begin{tabular}{|c|c|c|c|c|c|c|}
\hline Referencia & Pais & Título & $\begin{array}{l}\text { Diseño de } \\
\text { Estudio }\end{array}$ & Objetivo & Principales Resultados & Conclusiones \\
\hline $\begin{array}{l}\text { Lokade et } a l^{9} \text {, } \\
2019\end{array}$ & India & $\begin{array}{l}\text { Comparative evalua- } \\
\text { tion of clinical and } \\
\text { radiographic success of } \\
\text { three different lesion } \\
\text { sterilization and tissue } \\
\text { repair techniques as } \\
\text { treatment options in } \\
\text { primary molars requi- } \\
\text { ring pulpectomy: An in } \\
\text { vivo study } \\
\text { (Evaluación compara- } \\
\text { tiva del éxito clínico } \\
\text { y radiográfico de tres } \\
\text { técnicas diferentes } \\
\text { de esterilización de } \\
\text { lesiones y reparación de } \\
\text { tejidos como opciones } \\
\text { de tratamiento en } \\
\text { molares primarios que } \\
\text { requieren pulpectomía: } \\
\text { un estudio in vivo.) }\end{array}$ & $\begin{array}{l}\text { Ensayo clínico } \\
\text { aleatorizado }\end{array}$ & $\begin{array}{l}\text { Evaluar el éxito clínico y } \\
\text { radiográfico de tres técnicas } \\
\text { diferentes de LSTR*: } \\
\text { Grupo I: Extracción de la } \\
\text { pulpa coronaria solamente + } \\
\text { 3Mix MP*; } \\
\text { Grupo II: Extirpación tanto } \\
\text { del tejido pulpar coronal } \\
\text { como todo el tejido pulpar } \\
\text { radicular accesible + 3Mix } \\
\text { MP*; } \\
\text { Grupo III: Extracción sola- } \\
\text { mente de la pulpa coronária } \\
\text { + pasta CTZ. }\end{array}$ & $\begin{array}{l}\text { Los resultados mostraron que } \\
\text { las tasas de éxito clínico del } \\
\text { Grupo I, Grupo II y Grupo III } \\
\text { fueron } 90 \%, 90,5 \% \text { y } 81,8 \% \\
\text { respectivamente y las tasas de } \\
\text { éxito radiográfico fueron } 75 \% \text {, } \\
76,2 \% \text { y } 63,6 \% \text { respectiva- } \\
\text { mente después de } 12 \text { meses } \\
\text { de observación }\end{array}$ & $\begin{array}{l}\text { Sobre la base de las tasas } \\
\text { de éxito generales de las } \\
\text { tres técnicas LSTR, se puede } \\
\text { inferir el éxito clínico y el éxito } \\
\text { radiográfico siguiendo el orden } \\
\text { de ejecución: - } 3 \text { Mix-MP sin } \\
\text { extracción de pulpa radicular } \\
\text { = } 3 \text { Mix-MP con extracción de } \\
\text { pulpa radicular> pasta CTZ. }\end{array}$ \\
\hline $\begin{array}{l}\text { Souza et } a{ }^{10} \text {, } \\
2020\end{array}$ & Brasil & $\begin{array}{l}\text { Prevalence of enamel } \\
\text { defects in premolars } \\
\text { whose predecessors } \\
\text { were treated with } \\
\text { extractions or antibiotic } \\
\text { paste. } \\
\text { (Prevalencia de } \\
\text { defectos del esmalte } \\
\text { en premolares cuyos } \\
\text { predecesores fueron } \\
\text { tratados con extraccio- } \\
\text { nes o pasta antibiótica.) }\end{array}$ & $\begin{array}{l}\text { Estudio } \\
\text { Observacinal } \\
\text { Transversal }\end{array}$ & $\begin{array}{l}\text { Determinar la prevalencia de } \\
\text { defectos en el desarrollo del } \\
\text { esmalte (DDE) en premolares } \\
\text { cuyos predecesores tenían } \\
\text { antecedentes clínicos de: } \\
\text { Grupo CTZ -necrosis pulpar y } \\
\text { tratados con pasta CTZ; } \\
\text { Grupo E- necrosis pulpary } \\
\text { extraídos; } \\
\text { Grupo H- sanos. }\end{array}$ & $\begin{array}{l}\text { DDE estaban presentes en } \\
22,5 \% \text { de los } 1017 \text { premolares } \\
\text { incluidos, premolares en el } \\
\text { grupo } \mathrm{E} \text { tuvieron la mayor pro- } \\
\text { babilidad de } \mathrm{DDE}(\mathrm{OR}=3.52 \text {, } \\
95 \% \text { IC: } 2.29-5.40) \text { que los } \\
\text { del grupo CTZ (OR=2.45, } 95 \% \\
\text { IC: } 1.51-3.91 \text { ) y del grupo H } \\
\text { ( }<<0.01)\end{array}$ & $\begin{array}{l}\text { La prevalencia de DDE fue } \\
\text { mayor en los premolares cuyos } \\
\text { predecesores fueron extraídos } \\
\text { por necrosis pulpar seguidos de } \\
\text { los tratados con CTZ y los que } \\
\text { estaban sanos al momento de } \\
\text { la exfoliación }\end{array}$ \\
\hline
\end{tabular}

*LSTR - Lesion Sterilization and Tissue Repair

**3Mix MP- (metronidazol, minociclina, ciprofloxacina 1:1:1 y vehículos macrogol, propilenglicol 1:1)

\section{Discusión}

La pasta CTZ ha demostrado una buena actividad antimicrobiana sobre la polimicrobiota típica de las infecciones endodóncicas. ${ }^{12-15}$ Es importante considerar que los conductos radiculares de los dientes primarios con pulpas necróticas son infectados por una polimicrobiota con predominio de microorganismos anaerobios, bacilos pigmentados (porphyromonas y prevotella), algunos aerobios y estreptococos. ${ }^{19}$ Las especies frecuentemente encontradas son Porphyromonas gingivalis (73,3\%), Prevotella intermedia (6,7\%), Porphyromonas nigrescens (86,7\%), Fusobacterium alocis (73,3\%), y Treponema forsythia (26,7\%). ${ }^{20}$ El Enterococcus faecalis también ha sido detectado en dientes primarios necróticos y en infecciones endodóncicas persistentes por ser altamente resistente a los tratamientos. ${ }^{21}$ Este microorganismo puede colonizar áreas usualmente no alcanzadas por los métodos de desinfección. ${ }^{22}$ Por tanto, se justifica el uso de antibióticos combinados. $^{2,11,23}$

Con relación a los métodos de evaluación in vitro del efecto antimicrobiano, debemos 
considerar que la técnica de dilución en el medio de cultivo está indicada para sustancias solubles. En cuanto al método de difusión en agar, el grado de propagación de la sustancia interfiere con el resultado, lo que representa un problema al comparar materiales con diferentes grados de difusión. El método de contacto directo evalúa el efecto antimicrobiano de la sustancia en contacto directo con el microorganismo; de esta forma, no es influenciado por las variables mencionadas. ${ }^{24}$ En esta revisión narrativa, sólo dos estudios utilizaron esta metodología ${ }^{14,15}$ lo que podría considerarse una limitación de la presente revisión.

Dada la proximidad del diente sucesor, es importante que el material utilizado en dientes primarios sea sometido a pruebas in vitroyestudiospreclínicosinvivoparaevaluar su biocompatibilidad, y que los resultados pueden ser extrapolados en humanos. ${ }^{25} \mathrm{La}$ pasta CTZ fue considerada biocompatible por diferentes metodologías. ${ }^{16,17}$ Por el contrario, resultados insatisfactorios para citotoxicidad de la pasta CTZ en cultivo de osteoblastos humanos fueron obtenidos por Ern et al. ${ }^{18}$ Los autores atribuyeron los resultados a la presencia del eugenol y sugirieron, basándose en la literatura, que la citotoxicidad inicial podría disminuir con el tiempo sin la presencia de una respuesta inflamatoria relevante. Teniendo en cuenta estos resultados controversiales, se sugiere que deben realizarse más estudios preclínicos sobre este asunto.

Una posible desventaja clínica de la pasta CTZ sería la pigmentación de la corona del diente primario debido a su composición, lo que requiere precaución cuando se utiliza en dientes anteriores. ${ }^{5}$ Una alternativa para minimizar estos posibles efectos sería disminuir la proporción de los antimicrobianos tetraciclina y cloranfenicol en la composición de la pasta. ${ }^{13}$ Además, la presencia de tetraciclina en la composición de esta pasta podría generar inquietudes sobre el depósito de esta sustancia en estructuras dentales en desarrollo en pacientes pediátricos provocando posibles defectos en el desarrollo del esmalte en dientes permanentes. Sin embargo, se observó que la necrosis pulpar del molar primario, por sí sola, representa un factor de riesgo para la presentación de alteraciones del esmalte en los premolares sucesores. ${ }^{10}$ Se recomiendan más estudios que evalúen la aparición de cambios de color debido al uso de la pasta CTZ con menores proporciones de los antibióticos. ${ }^{13}$

La mayoría de los estudios clínicos mostraron altas tasas de éxito y la aparición de pocos eventos negativos. No obstante, siguieron varios criterios para evaluar el éxito clínico y radiográfico. Los criterios generales de éxito clínico más utilizados fueron: ausencia de dolor, edema, fístula o movilidad patológica; y criterios radiográficos incluyeron la reducción del área radiolúcida previamente observada, ${ }^{7-9}$ la ausencia de reabsorción patológica ${ }^{5,7-9}$ y ausencia de formación de radiotransparencia, en casos sin lesión radiográfica previa., 5,8,9 La Academia Americana de Odontología Pediátrica establece que después del tratamiento con técnica LSTR se debe observar la resolución de los signos y síntomas clínicos y los cambios radiográficos relacionados con el proceso infeccioso. ${ }^{28}$

De acuerdo con revisiones sistemáticas recientes, el uso del enfoque de esterilización de lesiones y reparación de tejidos (LSTR) en el tratamiento endodóncico de los dientes primarios ha mostrado ser un método eficaz ${ }^{27}$ y presenta una alta tasa de éxito clínico sobre todo se recomienda la técnica para reemplazar la 
pulpectomía para los dientes que presenten signos de reabsorción radicular que deben mantenerse en la cavidad bucal hasta por 12 meses. ${ }^{11}$ Las ventajas de utilizar la técnica sin instrumentación endodóncica y pasta CTZ en dientes primarios son relevantes. Es de fácil y rápido uso, no requiriendo de un profesional especializado, lo que la convierte en un material ideal no sólo para la atención de rutina en los servicios públicos,${ }^{6}$ sino también, para el tratamiento de pacientes no colaboradores que necesitan una intervención inmediata. ${ }^{4,8,13}$ En algunas situaciones, la permanencia del diente primario sin sintomatología clínica durante un año más, es suficiente para que el sucesor permanente pueda erupcionar sin la posibilidad de favorecer la aparición de maloclusiones en el paciente infantil. Se deben realizar estudios clínicos adicionales, de buena calidad metodológica, para que se genere evidencia más rica, que acrescente información relevante a la ya existente.

\section{Conclusión}

Aunque se necesitan más estudios que contribuyan a un mejor nivel de la evidencia, puede afirmase que la pasta CTZ es una alternativa para los casos de afectación pulpar en dientes primarios ya que la mayoría de estudios consideraron que la pasta CTZ es biocompatible, presenta actividad antimicrobiana satisfactoria y buenos resultados clínicos en el tratamiento endodóncico de dientes primarios mediante la técnica no instrumental, siendo su uso indicado en el área de odontopediatría, especialmente para el tratamiento de pacientes no colaboradores y en la atención pública.

\section{Referencias bibliográficas}

1. Ahmed, H M A. Anatomical challenges, electronic working length determination and current developments in root canal preparation of primary molar teeth. International Endodontic Journal. 2013;46:1011-1022.

2. Takushige T, Cruz EV, Asgor Moral A, Hoshino E. Endodontic treatment of primary teeth using a combination of antibacterial drugs. Int Endod J. 2004;37:132-8.

3. Cappiello J. Tratamentos pulpares em incisivos primários. Rev Asoc Odontol Argentina. 1964;52:139-45.

4. Cappiello J. Nuevos enfoques em odontologia infantil. Odontol Uruguai. 1967;23: 23-30.

5. Daher, A.; Viana, K. A.; Leles, C. R.; Costa, L. R. Ineffectiveness of Antibiotic-Based Pulpotomy for Primary Molars: a Survival Analysis. Pesquisa Brasileira Em Odontopediatria E Clinica Integrada. 2015;15(1):205215.

6. Siegl, R. M. C.; Lenzi, T. L.; Politano, G. T.; Benedetto, M. D. et al. Two endodontics techniques analysis in primary molars with fistula. RGO (Porto Alegre). 2015;63(2):187-194.

7. De Deus Moura, L. D. F. A.; De Lima, M. D. D. M.; Lima, C. C. B.; Machado, J. I. A. G. et al. Endodontic treatment of primary molars with antibiotic paste: A report of 38 cases. J Clin Pediatr Dent. 2016;40(3):175177.

8. Luengo-Fereira J, Ayala-Jiménez S, Carlos-Medrano LE, Toscano-García I, Anaya-Álvarez M. Clinical and Radiographic Evaluation of Formocresol and Chloramphenicol, Tetracycline and Zinc Oxide-Eugenol Antibiotic Paste in Primary Teeth Pulpotomies: 24 month follow up. J Clin Pediatr Dent. 2019;43(1):16-21.

9. Lokade A, Thakur S, Singhal P, Chauhan D, Jayam C. Comparative evaluation of clinical and radiographic success of three different lesion sterilization and tissue repair techniques as treatment options in primary molars requiring pulpectomy: An in vivo study. J Indian Soc Pedod Prev Dent. 2019;37(2):185-91.

10. Sousa HCS, Lima MDM, Lima CCB, Moura MS, Bandeira AVL, Deus Moura LFA. Prevalence of Enamel Defects in Premolars Whose Predecessors Were Treated with Extractions or Antibiotic Paste. Oral Health Prev Dent. 2020 Sep 4;18(1):793-798. doi: 10.3290/j.ohpd.a45083. PMID: 
11. Coll JA, Vargas K, Marghalani AA, et al. A Systematic Review and MetaAnalysis of Nonvital Pulp Therapy for Primary Teeth. Pediatr Dent 2020; 42(4):256-72.E11-E199

12. Gonçalves, S.G.; Silveira, R.G.; Miasato, J.M.; Oliveira, L.M.C.; Neves, A.A. Antimicrobial activity of endodontic pastes on microorganisms present in root canals of necrotic primary molars. Int J Clin Den. 2015;8(1): 213-223.

13. Oliveira S C de, Omena A L C S de, Lira G A de L, Ferreira I A, Imparato J C P, Calvo Ana F B. Do Different Proportions of Antibiotics in the CTZ Paste Interfere with the Antimicrobial Action? In Vitro Study. Pesqui. Bras. Odontopediatria Clín. Integr. [Internet]. 2019 [cited 2020 Dec 13]; 19: e4801. Available from: http://www.scielo.br/scielo.php?script=sci_arttext\&pid=S1983-46322019000100412\&lng=en. Epub Jan 13,2020. http://dx.doi.org/10.4034/pboci.2019.191.115.13.

14. Sancas MC, Souza ACL, Monteiro ASN, Pintor AVB, Duarte ML, Primo LSSG. Antimicrobial activity of antibiotic pastes used in pulp therapy through direct contact with a multispecies biofilm: A pilot study. Revista Científica do CRO-RJ (Rio de Janeiro Dental Journal) v. 5, n. 1, January - April, 2020.

15. Freire AR, Freire DEWG, Sousa SA, Serpa EM, Almeida LFD, Cavalcanti YW. Antibacterial and solubility analysis of experimental phytotherapeutic paste for endodontic treatment of primary teeth. Pesqui Bras Odontopediatria Clín Integr. 2021; 21:e0007 https://doi.org/10.1590/pboci.2021.005.

16. Lima, C. C. B.; Conde, A. M.; Rizzo, M. S.; Moura, R. D. et al. Biocompatibility of root filling pastes used in primary teeth. Int Endod J. 2015;48(5): 405-416.

17. Moura, L. F. A. D.; Lima,M. D. M.; Lima, C. C. B.; Bandeira, A. V. L. et al. Cellular profile of primary molars with pulp necrosis after treatment with antibiotic paste. Int J Exp Pathol. 2018;99(5):264-268.

18. Ern A, Pinto K, Silva E, Moreira, E 2020/03/31 1 In vitro cytotoxic effects of different endodontic pastes used in pediatric dentistry. Revista Brasileira de Odontologia. 2020.10.18363/rbo.v77.2020.e177119.

19. Silva LAB, Nelson-Filho P, Faria G, Souza-Gugelmin MCM, Ito IY. Bacterial profile in primary teeth with necrotic pulp and periapical lesion. Braz Dent J 2006; 17(2):144-8.20.

20. Gomes GB, Onofre SR, et al. An investigation of the presence of specific anaerobic species in necrotic primary teeth. Braz Oral Res. 2013;;27((2):):149--155.doi: 10.1590/S1806-83242013000100020.

21. Cogulu D, Uzel A, Oncag O, Aksoy SC, Eronat C. Detection of Enterococcus faecalis in Necrotic Teeth Root Canals by Culture and Polymerase Chain Reaction Methods. Eur J Dent. 2007 Oct;1(4):216-21. PMID: 19212470; PMCID: PMC2609913.

22. Zoletti GO, Pereira EM, Schuenck RP, Teixeira LM, Siqueira Jr JF, Santos KRN. Characterization of virulence factors and clonal diversity of Enterococcus faecalis isolates from treated dental root canals. Res Microbiol 2011; (2):151-8. https://doi.org/10.1016/j.resmic.2010.09.018

23. Parasuraman RV, Muljibhai BS. 3Mix-MP in endodontics - An overview. JDMS 2012;3(1):36-45.

24. Estrela C, Bammann LL, Pimenta FC, Pécora JD. Control of microorganisms in vitro by calcium hydroxide pastes. Int Endod J 2000;34:416-418.

25. Hauman CHJ, Love RM. Biocompatibility of dental materials used in contemporary endodontic therapy: a review. Part 2. Root-canal filling materials. Int Endod J. 2003;36:147-60.

26. American Academy of Pediatric Dentistry. Pulp treatment for primary and immature permanent teeth. The Reference Manual of Pediatric Dentistry. Chicago, 111.: American Academy of Pediatric Dentistry; 2019:353-61.

27. Duarte ML, Pires PM, Ferreira DM, Pintor AVB, Neves AA, Maia LC, Primo LG . Is there evidence for the use of lesion sterilization and tissue repair therapy in the endodontic treatment of primary teeth? A systematic review and meta-analyses [published online ahead of print, 2020 Jul 14]. Clin Oral Investig. 2020;10.1007/s00784-020-03415-0. doi:10.1007/s00784-020-03415-0

Recibido: $18 / 01 / 21$

Aceptado: $12 / 09 / 21$

Correspondencia: Ana Paula Portes Zeno, correo: anappzeno@gmail.com 